\title{
Spin-helix-driven insulating phase in two-dimensional lattice
}

\author{
HaRu K. Park, ${ }^{*}$ Hyeok-Jun Yang, ${ }^{\dagger}$ and SungBin Lee ${ }^{\ddagger}$ \\ Department of Physics, Korea Advanced Institute of Science and Technology, Daejeon 34141, Korea
}

(Received 2 December 2019; revised 29 June 2020; accepted 1 July 2020; published 24 September 2020)

\begin{abstract}
Motivated by emergent SU(2) symmetry in the spin-orbit-coupled system, we study the spin-helix-driven insulating phase in a two-dimensional lattice. When both Rashba and Dresselhaus spin-orbit couplings are present, the perfect Fermi-surface nesting occurs at a special condition depending on the lattice geometry. In this case, the energies of spin up at any wave vector $\vec{k}$ are equivalent to the ones of spin down at $\vec{k}+\vec{Q}$ with the shifting wave vector $\vec{Q}$. Thus, the system stabilizes the magnetic insulator with spiral-like magnetic ordering even in the presence of tiny electron-electron interaction where the magnetic ordering wave vector is proportional to $\vec{Q}$. We first show the condition for the existence of the shifting wave vector in a general lattice model and emergent SU(2) symmetry in the spin-orbit-coupled system. Then, we exemplify this in a square lattice at half filling and discuss the insulating phase with (non)coplanar spin density wave and charge order. Our study emphasizes different possible types of two-dimensional magnetic materials that can be applicable to various van der Waals materials and their heterostructures with the control of electric field, strain, and pressure.
\end{abstract}

DOI: 10.1103/PhysRevResearch.2.033487

\section{INTRODUCTION}

Spin SU(2) symmetry invariance with respect to electron spin rotation is an important quantity giving conservation of spin polarization in the system. When spin and orbital degrees of freedom are coupled, however, such SU(2) symmetry is generally broken, and thus spin polarization is no longer a good quantum number. Particularly for a two-dimensional system including surfaces, two different types of spin-orbit couplings (SOCs) are mainly addressed due to broken inversion symmetries, Rashba and Dresselhaus spin-orbit couplings. The Rashba effect originates from the effective electric field at the surface or interface of crystal structures (SIA), whereas the Dresselhaus effect comes from the bulk inversion asymmetry (BIA). Their measurement and controllability have been widely studied in quantum wells for several decades [1]. More recently, the possible creation of such interactions has been explored even in optical lattices [2,3].

Despite general consensus that the spin-orbit effect breaks spin SU(2) symmetry, some exotic cases are known to host emergent symmetry higher than $\mathrm{SU}(2)$, such as $\mathrm{SU}(4)$, as a result of strong spin and orbital coupling [4]. Moreover, it has been pointed out that well control of Rashba and Dresselhaus effects can give rise to not only U(1) symmetry [5], but also emergent SU(2) symmetry [6]. In particular, when Rashba and Dresselhaus SOCs are equal in two-dimensional electron

\footnotetext{
*p_haru@kaist.ac.kr

†yang267814@kaist.ac.kr

${ }^{\ddagger}$ sungbin@kaist.ac.kr
}

Published by the American Physical Society under the terms of the Creative Commons Attribution 4.0 International license. Further distribution of this work must maintain attribution to the author(s) and the published article's title, journal citation, and DOI. gas, it can be exactly mapped into the Dresselhaus [110] model which describes a quantum well grown along the [110] direction $[7,8]$. Then the effective magnetic field via SOC is unidirectionally induced, and thus spin polarization of a helical mode is conserved along that special direction, leading to a new type of SU(2) symmetry. Such emergent SU(2) symmetry with persistent spin helix has become one of the central topics of experimental realization in semiconductor quantum wells and their direct observations $[2,3,9,10]$.

In general, microscopic control of SOC has been regarded as a very challenging task. In the two-dimensional electron gas of semiconductors, however, possible control of SOC is studied via the gate electric field or tunneling current, etc. [11-14]. Moreover, at the interface of oxide heterostructures and van der Waals heterostructures, the realization of a giant SOC with heavy ions is extensively studied both in experimental and theoretical aspects [15-19]. Such controllability and enhancement of SOC have received much attention in pursuing control of spin precession potentially related to spintronic devices and quantum computations. Along with controlling SOCs mentioned above, the interplay of electron correlation can also give rise to new emergent phenomena. Especially in oxide heterostructures and transition-metal chalcogenide heterostructures, both SOC and electron correlation play an important role.

In this paper, taking into account the possible controllability of SOC in two-dimensional heterostructures, we discuss the magnetic insulator induced by the interplay of SOC and electron correlation. We extend the analysis of twodimensional free gas [6] and determine the ratio $\delta$ between Rashba and Dresselhaus SOC strengths specific to the lattice geometry to generate a spin helix. Our work is not simply the generalization of free gas, but rather suggests a different type of magnetic insulator characterized by the shifting wave vector $\vec{Q}$ satisfying $\epsilon_{\vec{k}+\vec{Q}, \uparrow}=\epsilon_{\vec{k}, \downarrow}$. Two types of magnetic instabilities are considered, where one is intrinsic to the system [20] 
and the other is induced by tuned SOCs. Consequently, an infinitesimal electron interaction drives the ground state to develop a long-range magnetic order which is absent in the free gas.

We demonstrate our scenario through the Hubbard model on a half-filled square lattice. To investigate the magnetic instability, we evaluate the susceptibility and a phase diagram as varying the Coulomb repulsion $U$ and SOC strength ratio $\delta$. Based on the noninteracting limit, the magnetic instability associated with the nesting allows us to employ the HartreeFock approximation in the small- $U$ regime [21,22]. We find a magnetic insulator stabilized by a noncoplanar spin density wave in addition to charge order. By controlling the relative ratio between Rashba and Dresselhaus SOCs, we discuss the possible phase diagram for a metal-insulator transition and generalize to other lattices. Our work introduces a mechanism of two-dimensional magnetic insulators applicable to many transition-metal oxides and chalcogenides, and their heterostructures.

\section{GENERAL LATTICE MODEL AND SHIFTING WAVE VECTOR}

On a two-dimensional lattice, we consider the Hubbard model with SOCs as

$$
\begin{gathered}
H_{t, U}=-\sum_{i, j, \alpha} t_{i j} c_{i, \alpha}^{\dagger} c_{j, \alpha}+\text { H.c. }+U \sum_{i} n_{i \uparrow} n_{i \downarrow}, \\
H_{\mathrm{soc}}=i \sum_{i, j, \alpha, \beta} s_{i j}\left(\hat{d}_{i j} \cdot \vec{\sigma}\right)_{\alpha \beta} c_{i, \alpha}^{\dagger} c_{j, \beta}+\text { H.c. }
\end{gathered}
$$

where $c_{i, \sigma}^{\dagger}, c_{i, \sigma}$, and $n_{i \sigma}$ are the electron creation, annihilation, and number operators at site $i$ with spin $\alpha, \beta \in(\uparrow, \downarrow)$, respectively. In Eqs. (1) and (2), the lattice sites $i, j$ are not restricted to the nearest neighbors and the unit vector $\hat{d}_{i j}$ characterizes the SOC with the Pauli matrices $\vec{\sigma}=\left(\sigma^{x}, \sigma^{y}, \sigma^{z}\right)$.

Let us first consider the noninteracting system $(U=0)$ and examine the Fermi-surface properties. We focus on the case when the magnitudes of Rashba and Dresselhaus SOCs satisfy a particular ratio specific to the lattice geometry. (We will show this later.) Then, the SOC in Eq. (2) after a proper spin rotation can be written as

$$
\tilde{H}_{\mathrm{soc}}=i \sum_{i, j, \sigma_{z}} \sigma^{z} s_{i j}\left(c_{i, \sigma_{z}}^{\dagger} c_{j, \sigma_{z}}-c_{j, \sigma_{z}}^{\dagger} c_{i, \sigma_{z}}\right) .
$$

In this limit, our aim is to demonstrate the existence of the shifting wave vector $\vec{Q}$, which is defined to satisfy the shifting condition $\epsilon_{\vec{k}+\vec{Q}, \uparrow}=\epsilon_{\vec{k}, \downarrow}$, where $\epsilon_{\vec{k}, \sigma^{z}}$ is the energy dispersion in $\vec{k}$ space with spin $\uparrow, \downarrow$. For a given hopping magnitude $t_{i j}$ and the SOC strength $s_{i j}$, the shifting wave vector $\vec{Q}$ always exists if they meet

$$
s_{i j}=t_{i j} \tan \left[\vec{Q} \cdot\left(\vec{x}_{i}-\vec{x}_{j}\right) / 2\right] .
$$

This indicates that it is possible to choose the appropriate SOC strength $s_{i j}$ for an arbitrary vector $\vec{Q}$ and the hopping strength $t_{i j}$. Generically, it can be shown that a two-level Hamiltonian with a form $H_{\sigma^{z}}(k)=h(k) I_{2 \times 2}+g(k) \sigma^{z}$ admits these shifting conditions (see the Supplemental Material [23]). To study the nesting property, we define the spin operators

$$
\begin{aligned}
S_{\vec{k}, \vec{Q}}^{-} & =c_{\vec{k}, \downarrow}^{\dagger} c_{\vec{k}+\vec{Q}, \uparrow}, \quad S_{\vec{k}, \vec{Q}}^{+}=c_{\vec{k}+\vec{Q}, \uparrow}^{\dagger} c_{\vec{k}, \downarrow}, \\
S_{\vec{k}, 0}^{z} & =c_{\vec{k}, \uparrow}^{\dagger} c_{\vec{k}, \uparrow}-c_{\vec{k}, \downarrow}^{\dagger} c_{\vec{k}, \downarrow},
\end{aligned}
$$

obeying the standard $\mathrm{SU}(2)$ commutation relations $\left[S_{\vec{k}, 0}^{z}, S_{\vec{k}, \vec{Q}}^{ \pm}\right]= \pm 2 S_{\vec{k}, \vec{Q}}^{ \pm}\left[S_{\vec{k}, \vec{Q}}^{+}, S_{\vec{k}, \vec{Q}}^{-}\right]=S_{\vec{k}, 0}^{z}$. Since they commute with the Hamiltonian $\left[H, S_{\vec{k}, \vec{Q}}^{ \pm}\right]=\left[H, S_{\vec{k}, 0}^{z}\right]=0$, the emergent $\mathrm{SU}(2)$ symmetry enables us to manipulate Eq. (5) for the magnetic order parameters.

\section{PERFECT NESTING IN HALF-FILLED SQUARE LATTICE}

Now suppose the square lattice and the nearest-neighbor hoppings $t_{i j}=t$ only. The shifting condition given by Eq. (4) requires $s_{i j}= \pm s_{x(y)}$ for $\vec{r}_{i}=\vec{r}_{j} \pm \hat{x}(\hat{y})$, and $s_{i j}=0$ otherwise. We can choose $\left(s_{x}, s_{y}\right)=t\left[\tan \left(Q_{x} / 2\right), \tan \left(Q_{y} / 2\right)\right]$ for a given shifting vector $\vec{Q}=\left(Q_{x}, Q_{y}\right)$. Inversely, the shifting vector $\vec{Q}=2\left[\tan ^{-1}\left(s_{x} / t\right), \tan ^{-1}\left(s_{y} / t\right)\right]$ can be designated for arbitrary strength $\left(s_{x}, s_{y}\right)$. With the nearest-neighbor SOCs, Eq. (2) is decomposed into Rashba and Dresselhaus SOCs,

$$
\begin{aligned}
& H_{R}=i s_{R} \sum_{\vec{r}}\left(-\sigma_{\alpha \beta}^{y} c_{\vec{r}, \alpha}^{\dagger} c_{\vec{r}+\hat{x}, \beta}+\sigma_{\alpha \beta}^{x} c_{\vec{r}, \alpha}^{\dagger} c_{\vec{r}+\hat{y}, \beta}\right)+\text { H.c., } \\
& H_{D}=i s_{D} \sum_{\vec{r}}\left(\sigma_{\alpha \beta}^{x} c_{\vec{r}, \alpha}^{\dagger} c_{\vec{r}+\hat{x}, \beta}-\sigma_{\alpha \beta}^{y} c_{\vec{r}, \alpha}^{\dagger} c_{\vec{r}+\hat{y}, \beta}\right)+\text { H.c. }
\end{aligned}
$$

The prerequisite for the shifting condition given by Eq. (3) can be attained when $s_{R}=s_{D} \equiv s / \sqrt{2}$, whose ratio $s_{R} / s_{D}=$ 1 is specific to the square lattice. After $\pi / 2$ spin rotation along the $(\hat{x}+\hat{y})$ axis, $H_{R}+H_{D}$ is rearranged into the form of Eq. (3) with the dispersion $\epsilon_{\vec{k}, \sigma^{z}}=-2 t\left[\cos \left(k_{x}\right)+\cos \left(k_{y}\right)\right]+$ $2 s \sigma^{z}\left[\sin \left(k_{x}\right)+\sin \left(k_{y}\right)\right]$. It satisfies the shifting property $\epsilon_{\vec{k}+\vec{Q}, \uparrow}=\epsilon_{\vec{k}, \downarrow}$ in the rotated spin basis, with the shifting wave vector $\vec{Q}=2 \tan ^{-1}(s / t)(1,1)$. For a small SOC, this agrees with the results of free electron gas since the hopping amplitude is inversely proportional to the effective mass [6].

Taking into account the shifting property in the case $s_{R}=s_{D}$, the Fermi surfaces for up/down spins are perfectly split, as shown in Fig. 1(a). This generates the nesting between Fermi surfaces for opposite spins with the wave vectors $\vec{K}_{ \pm}=(\pi, \pi) \pm \vec{Q}$. Furthermore, due to the shape of the Fermi surface itself at half filling, there is additional nesting for each Fermi surface with the wave vector $\vec{K}^{*}=(\pi, \pi)$. Having these in mind, one expects the magnetic instabilities near $\vec{K}_{ \pm}$and $\vec{K}^{*}$. The spin susceptibility at a wave vector $\vec{q}$ is related to the scattering amplitude between the filled and empty states near the Fermi level separated by $\vec{q}$, $\chi^{\mu}(\vec{q})=-\sum_{\vec{k}, \sigma}\left[f\left(\epsilon_{\vec{k}, \sigma}\right)-f\left(\epsilon_{\vec{k}+\vec{q}, \sigma^{\prime}}\right)\right] /\left(\epsilon_{\vec{k}, \sigma}-\epsilon_{\vec{k}+\vec{q}, \sigma^{\prime}}\right)$, with $\mu=\perp$, $\|$ [20]. Here, $\sigma=-\sigma^{\prime}$ for $\chi^{\perp}$, whereas $\sigma=\sigma^{\prime}$ for $\chi^{\|}$ and $f\left(\epsilon_{\vec{k}, \sigma}\right)$ is the occupation number of the energy $\epsilon_{\vec{k}, \sigma}$. It is noteworthy that the instability of $\chi^{\|}(\vec{q})$ near $\vec{K}^{*}$ is special for the square lattice at half filling, while the behavior of $\chi^{\perp}(\vec{q})$ near $\vec{K}_{ \pm}$is comprehensive regardless of the lattice geometry and the electronic filling. 


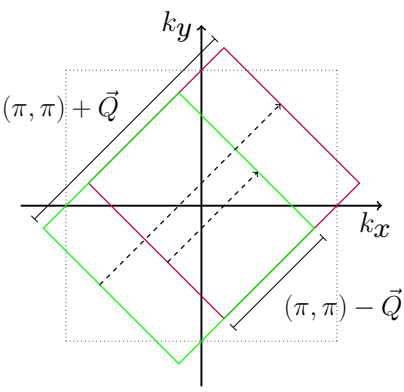

(a)

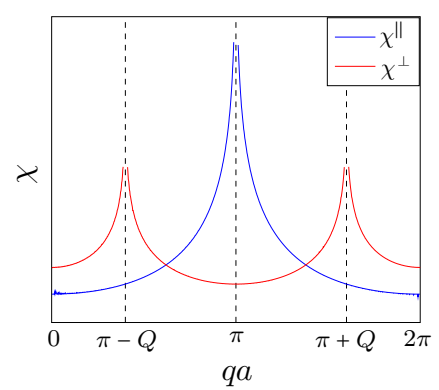

(b)
FIG. 1. Fermi surface and spin susceptibilities for square lattice at half filling with equal magnitudes of Rashba and Dresselhaus spinorbit couplings. (a) The split Fermi surfaces with the shifting vector $\vec{Q}=(0.6 \pi, 0.6 \pi)$. With a proper spin rotation, the green and purple colors represent the Fermi surfaces for up- and down-spin electrons, respectively. The dashed lines correspond to the wave vectors $\vec{K}_{ \pm}$ for perfect Fermi-surface nesting. (b) Spin susceptibilities $\chi^{\|}(\vec{q})$ and $\chi^{\perp}(\vec{q})$ for $\vec{q}=(q, q)$. The specific choice of $\vec{Q}$ only shifts the peak and does not affect the overall behavior.

Along the line $k_{x}=k_{y}$ in $\vec{k}$ space, the spin susceptibility $\chi^{\|}(q, q)$ is approximately evaluated as $\chi^{\|}(q, q) \sim F(q)$, where $F(q)=\frac{1}{\sin (q / 2)} \ln \left[\frac{1+\sin (q / 2)}{1-\sin (q / 2)}\right]$. The divergence of $F(x)$ at $x=\pi$ represents the magnetic instability at $\vec{q}=\vec{K}^{*}$. Similarly, one can estimate $\chi^{\perp}(q, q)$ as $\chi^{\perp}(q, q) \sim F(q+Q)$ for $0<q<\pi$, and $\chi^{\perp}(q, q) \sim F(q-Q)$ for $\pi<q<2 \pi$. This implies that the perfect Fermi-surface nestings at $\vec{q}=\vec{K}^{*}$ and $\vec{K}_{ \pm}$are associated with the spontaneous magnetization $\left\langle S_{\vec{K}^{*}}^{\|}\right\rangle$and $\left\langle S_{\vec{K}_{ \pm}}^{\perp}\right\rangle$, respectively. As the magnitude of SOC, $s$, varies, the peak of $\chi^{\perp}(\vec{q})$ moves with the shifting vector $\vec{Q}$, while $\chi^{\|}(\vec{q})$ does not. In Fig. 1(b), the spin susceptibilities are numerically evaluated with $Q=0.6 \pi$ as functions of $\vec{q}=$ $(q, q)$. Indeed, the magnetic susceptibilities $\chi^{\perp}(\vec{q})$ and $\chi^{\|}(\vec{q})$ diverge at $K_{ \pm}$and $K^{*}$, respectively. The overall weight near the peak reflects the density of states in which the nesting happens close to the Fermi surfaces. The magnetic susceptibility $\left|\chi^{\|}\left(\vec{q} \approx \vec{K}^{*}\right)\right|$ originates from the density of states for both spins, while $\left|\chi^{\perp}\left(\vec{q} \approx \vec{K}_{ \pm}\right)\right|$is proportional to the ones for each spin, which explains half of the magnitude compared to $\left|\chi^{\|}\left(\vec{q} \approx \vec{K}^{*}\right)\right|$.

\section{MAGNETIC INSULATOR WITH SPIN-HELIX ORDERING}

With the magnetic instabilities at the wave vectors $\vec{K}_{ \pm}$, $\vec{K}^{*}$, the ground state is expected to stabilize the spin and charge orderings with electron interaction $U$. We consider the quartic term $U n_{i \uparrow} n_{i \downarrow}$ with the Hartree-Fock approximation, keeping the dominant orderings $\left\langle S_{\vec{K}_{ \pm}}^{\perp}\right\rangle,\left\langle S_{\vec{K}^{*}}^{\|}\right\rangle$, and $\left\langle n_{\vec{K}^{*}}\right\rangle$ only. We further generalize our analysis when the ratio of Rashba SOC to Dresselhaus SOC deviates from the emergent $\mathrm{SU}(2)$ symmetric point, $\delta \equiv s_{R}-s_{D} \neq 0$. A small but finite $\delta$ implies the collapse of the perfect Fermi-surface nesting. The polarized spin identifying the band dispersion is now momentum dependent, $\epsilon_{\vec{k}, \sigma^{z}} \rightarrow \epsilon_{\vec{k}, \tilde{\sigma}^{z}\left(\theta_{\vec{k}}\right)}$ (see the Supplemental Material [23] for details.) Unlike the case of perfect Fermi-

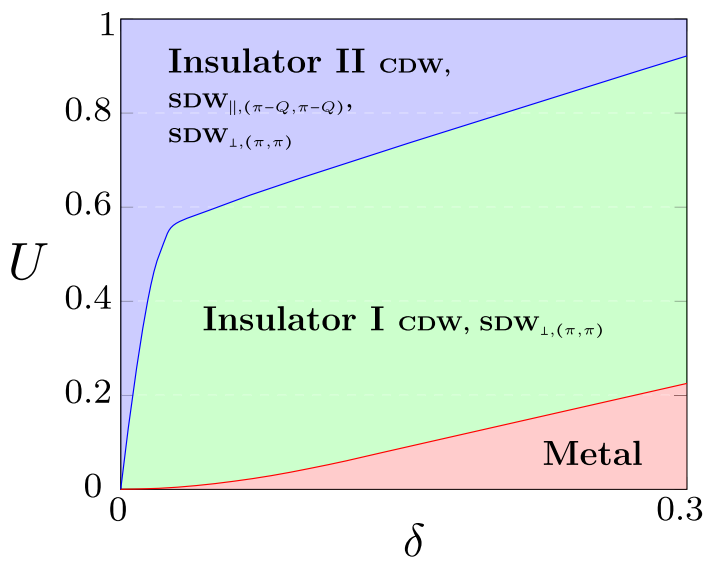

FIG. 2. The phase diagram as functions of electron interaction $U$ and $\delta=s_{R}-s_{D}$. Insulator I: The charge density wave and spin density wave exist with the wave vector $\vec{K}^{*}=(\pi, \pi)$, where the collinear magnetic moments are (anti)parallel to the $\hat{x}+\hat{y}$ direction. Insulator II: In addition to the charge and spin density waves of Insulator I, spiral magnetic order with the wave vector $\vec{K}_{ \pm}=(\pi \pm$ $Q, \pi \pm Q$ ) is further stabilized on the plane perpendicular to $\hat{x}+\hat{y}$, resulting in noncoplanar spin states. See Fig. 3 for details of the charge and spin density waves.

surface nesting, the critical value of electron interaction $U$ is required to stabilize the magnetic order when $\delta \neq 0$. Similar to the Stoner criterion for a ferromagnet, the critical values of $U$ are inversely proportional to the maximum peak of the magnetic susceptibilities $\chi_{\delta}^{\perp}\left(\vec{K}_{ \pm}\right)$and $\chi_{\delta}^{\|}\left(\vec{K}^{*}\right)$, which are damped for $\delta \neq 0$.

Figure 2 represents the phase diagram as functions of electron interaction $U$ and $\delta=s_{R}-s_{D}$. With increasing electron interaction $U$, there exists the phase transition from a metal to insulator I phase, where both the spin density wave (SDW) and charge density wave (CDW) are stabilized with the wave vector $\vec{K}^{*}$. Further increasing electron interaction

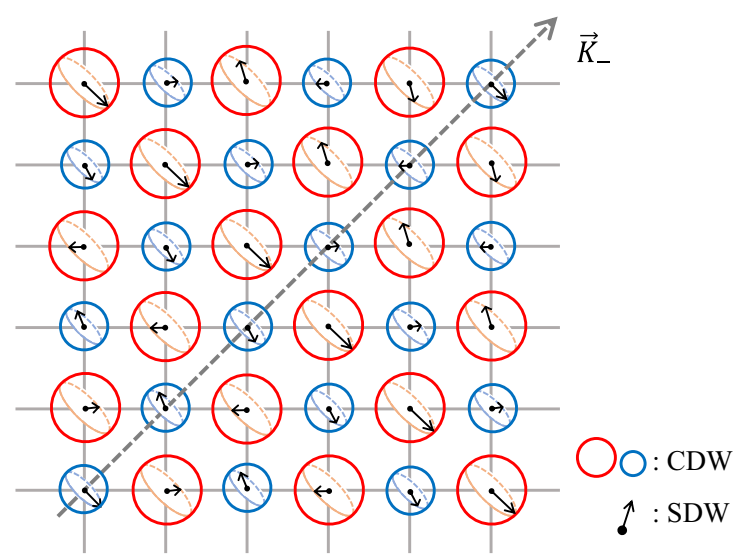

FIG. 3. Schematic depiction of the insulator II phase with $\vec{Q}=$ $(0.6 \pi, 0.6 \pi)$. The circle size and caged arrow represent the amount of charge order $\left\langle n_{\vec{K}^{*}}\right\rangle$ and (in-plane perpendicular $\hat{x}+\hat{y}$ axis) magnetic order $\left\langle S_{\vec{K}_{-}}^{\perp}\right\rangle$, respectively. The out-of plane magnetization $\left\langle S_{\vec{K}^{*}}^{\|}\right\rangle$, which is (anti)parallel to the $\hat{x}+\hat{y}$ axis, is built in the (red) blue circle with magnitude proportional to the circle size. 


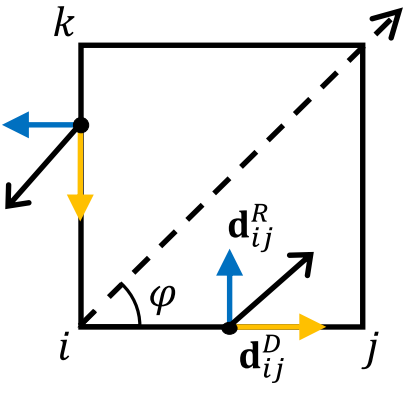

(a)

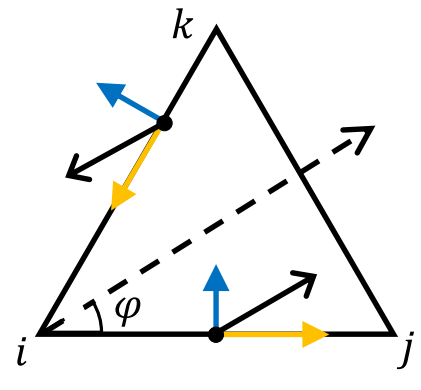

(b)
FIG. 4. The characterizing SOC vector $s_{i j} \hat{d}_{i j}=\mathbf{d}_{i j}^{D}+\mathbf{d}_{i j}^{R}$ on the link $i j$ and the mirror reflected one $s_{i k} \hat{d}_{i k}$ about the mirror plane (dashed line) on the (a) square lattice and (b) triangular lattice. The SOC vector $s_{i j} \hat{d}_{i j}$ (black arrow) is decomposed as a sum of the Dresselhaus (yellow arrow) and Rashba (blue arrow) SOCs.

$U$, the system develops the insulator II phase and stabilizes the SDW with the wave vector $\vec{K}^{ \pm}$, in addition to CDW and SDW with the wave vector $\vec{K}^{*}$. Thus, in this regime, the system develops the noncoplanar magnetic ordering both with $\left\langle S^{\perp}(\vec{r})\right\rangle \sim \cos \left(\vec{K}^{ \pm} \cdot \vec{r}\right)$ and $\left\langle S^{\|}(\vec{r})\right\rangle \sim \cos \left(\vec{K}^{*} \cdot \vec{r}\right)$ in addition to the charge order. Figure 3 shows the noncoplanar magnetic order along with charge order specifically with $\vec{K}^{-}=$ $(0.4 \pi, 0.4 \pi)$ and $\vec{K}^{*}$. It is worthwhile to note that the insulator I phase is specific to the square lattice at half filling, while the generic metal-insulator phase transition will occur between the metal and the insulator II regime.

\section{DISCUSSION}

We have studied the possible spin-helix-driven insulating phases, exemplifying the case of a square lattice at half filling. In this case, the shifting vector exists with an emergent SU(2) symmetry when Rashba and Dresselhaus SOCs take equal magnitudes. However, as mentioned above, our argument generally holds for other lattice geometries by controlling SOCs in different ratios. This implies that the transition to magnetic insulator II in Fig. 2 occurs quite generally regardless of the shape of the Fermi surface, while insulator I is specific to the square lattice at half filling. Thus, here we briefly discuss how to obtain emergent SU(2) symmetry for the case of a triangular lattice and compare with the case of a square lattice.

In order to obtain the emergent SU(2) symmetry, combinations of SOCs should be the form of Eq. (3) after a proper spin rotation. Equation (3) can be constructed when the characterizing vector $\hat{d}_{i j}$ in Eq. (2) is aligned in a collinear fashion. The characterizing vector $s_{i j} \hat{d}_{i j}$ in Eq. (2) is decomposed as a sum of the Dresselhaus and Rashba SOCs, $s_{i j} \hat{d}_{i j}=\mathbf{d}_{i j}^{D}+\mathbf{d}_{i j}^{R}=$ $d_{i j}^{D} \hat{x}+d_{i j}^{R} \hat{y}$, in the coordinate whose $\hat{x}$ axis is towards the link $i j$. In the presence of the translational invariance, all vectors $s_{i j} \hat{d}_{i j}$ on other links can be obtained by the lattice translations combined with the mirror reflection. Here, for simplicity, we consider the mirror planes (containing dashed lines in Fig. 4), which bisect the acute angles between two adjacent links. To ensure that SOC vector $\hat{d}_{i j}$ is collinear with the mirror reflected one $\hat{d}_{i k}$, the requirement is $d_{i j}^{R} / d_{i j}^{D}=\tan (\varphi)$ or $d_{i j}^{R} / d_{i j}^{D}=\tan (\pi / 2+\varphi)$, where $\varphi$ is the angle between the mirror plane and the lattice link. This angle corresponds to the half acute angle between two adjacent links emanated from $i$ to $j$ and $k$. For example, the square lattice with $\varphi=\pi / 4$ demands $\left|\mathbf{d}_{i j}^{R}\right|=\left|\mathbf{d}_{i j}^{D}\right|$ on every link.

Now, we move on to the triangular lattice. Unlike the square lattice, there is a perpendicular link $j k$ which is reflected in itself, allowing $s_{j k} \hat{d}_{j k}=\mathbf{d}_{j k}^{D}$ only and $\mathbf{d}_{j k}^{R}=0$. All SOC vectors $s_{j k} \hat{d}_{j k}$ on these perpendicular links keep arbitrary magnitudes of $\mathbf{d}_{j k}^{D}$ unless constrained by other symmetries. Meanwhile, on the link $i j$ to be reflected in $i k$, the SOC condition becomes $\mathbf{d}_{i j}^{R}=\mathbf{d}_{i j}^{D} / \sqrt{3}$ or $\mathbf{d}_{i j}^{R}=-\sqrt{3} \mathbf{d}_{i j}^{D}$ since $\varphi=$ $\pi / 6$. On the perpendicular link $j k, \mathbf{d}_{j k}^{R}$ is forbidden in both cases, while arbitrary $\mathbf{d}_{j k}^{D}$ is allowed only in the latter case $\mathbf{d}_{i j}^{R}=-\sqrt{3} \mathbf{d}_{i j}^{D}$. In this manner, one can readily generalize the nesting condition with the emergent SU(2) symmetry by controlling the SOCs ratio $\delta$ for a generic two-dimensional lattice.

\section{CONCLUSION}

Perfect Fermi-surface nesting and emergent SU(2) symmetry are present exactly at fine tuning of Rashba and Dresselhaus SOCs to satisfy the special ratio, which is very challenging. However, as we have discussed above, Fermi-surface nesting induced by the combination of SOCs is still present in the vicinity of such fine tuning, even away from the exact ratio between SOCs. In principle, the effective electric fields for both in plane and out of plane can be controlled via applying gate voltage, pressure, or strain effect due to the substrate. Thus, at surfaces or interfaces of heterostructures, one may be able to access the parameter range where the system stabilizes the spin-helix-driven insulating phase due to the combination of SOCs. Our theoretical study introduces a way to approach magnetic insulators induced by the SOC effect in two-dimensional materials and their heterostructures. In the future, it would be interesting to explore the experimental controllability of SOCs in two-dimensional materials, especially the transition-metal chalcogenide series and oxide heterostructures, and look for magnetic insulators and their phase transitions.

\section{ACKNOWLEDGMENTS}

H.R.K.P., H.-J.Y., and S.B.L. are supported by the KAIST startup and National Research Foundation Grant No. NRF2017R1A2B4008097.
[1] J. D. Koralek, C. P. Weber, J. Orenstein, B. A. Bernevig, S.-C. Zhang, S. Mack, and D. Awschalom, Nature (London) 458, 610 (2009).

\begin{tabular}{|c|c|c|c|}
\hline $\begin{array}{l}\text { Y.-J. Lin, } \\
\text { Spielman, } \\
(2011) \text {. }\end{array}$ & $\begin{array}{l}\mathrm{K} . \\
\text { Nature }\end{array}$ & $\begin{array}{r}\text { Jiménez-García, } \\
\text { (London) }\end{array}$ & $\begin{array}{l}\text { and } \\
471,\end{array}$ \\
\hline
\end{tabular}


[3] F. Grusdt, T. Li, I. Bloch, and E. Demler, Phys. Rev. A 95, 063617 (2017).

[4] M. G. Yamada, M. Oshikawa, and G. Jackeli, Phys. Rev. Lett. 121, 097201 (2018).

[5] J. Schliemann, J. C. Egues, and D. Loss, Phys. Rev. Lett. 90, 146801 (2003).

[6] B. A. Bernevig, J. Orenstein, and S.-C. Zhang, Phys. Rev. Lett. 97, 236601 (2006).

[7] Y. Ohno, R. Terauchi, T. Adachi, F. Matsukura, and H. Ohno, Phys. Rev. Lett. 83, 4196 (1999).

[8] J. Alicea, Phys. Rev. B 81, 125318 (2010).

[9] M. Walser, C. Reichl, W. Wegscheider, and G. Salis, Nat. Phys. 8, 757 (2012).

[10] F. Dettwiler, J. Fu, S. Mack, P. J. Weigele, J. C. Egues, D. D. Awschalom, and D. M. Zumbühl, Phys. Rev. X 7, 031010 (2017).

[11] J. Nitta, T. Akazaki, H. Takayanagi, and T. Enoki, Phys. Rev. Lett. 78, 1335 (1997).

[12] G. Engels, J. Lange, T. Schäpers, and H. Lüth, Phys. Rev. B 55, R1958(R) (1997).

[13] T. Koga, J. Nitta, T. Akazaki, and H. Takayanagi, Phys. Rev. Lett. 89, 046801 (2002).
[14] L. Chen, M. Gmitra, M. Vogel, R. Islinger, M. Kronseder, D. Schuh, D. Bougeard, J. Fabian, D. Weiss, and C. H. Back, arXiv:1803.01656.

[15] M. Kim, J. Ihm, and S. B. Chung, Phys. Rev. B 94, 115431 (2016).

[16] A. Manchon, H. C. Koo, J. Nitta, S. Frolov, and R. Duine, Nat. Mater. 14, 871 (2015).

[17] K. Ishizaka, M. Bahramy, H. Murakawa, M. Sakano, T. Shimojima, T. Sonobe, K. Koizumi, S. Shin, H. Miyahara, A. Kimura et al., Nat. Mater. 10, 521 (2011).

[18] Z. Zhong, L. Si, Q. Zhang, W.-G. Yin, S. Yunoki, and K. Held, Adv. Mater. Interfaces 2, 1400445 (2015).

[19] A. D. Caviglia, M. Gabay, S. Gariglio, N. Reyren, C. Cancellieri, and J.-M. Triscone, Phys. Rev. Lett. 104, 126803 (2010).

[20] P. Fazekas, Lecture Notes on Electron Correlation and Magnetism (World Scientific, 1999).

[21] A. W. Overhauser, Phys. Rev. 128, 1437 (1962).

[22] V. Bach and J. Poelchau, J. Math. Phys. 38, 2072 (1997).

[23] See Supplemental Material at http://link.aps.org/supplemental/ 10.1103/PhysRevResearch.2.033487 for the calculation of shifting function and $\delta \neq 0$-spin susceptibility. 